\title{
Multilinear Fourier multipliers on variable Lebesgue spaces
}

Jineng Ren* and Wenchang Sun

\section{"Correspondence:}

renjineng@mail.nankai.edu.cn School of Mathematical Sciences and LPMC, Nankai University,

Tianjin, 300071, China

\begin{abstract}
In this paper, we study the properties of a bilinear multiplier space. We give a necessary condition for a continuous bounded function to be a bilinear multiplier on variable exponent Lebesgue spaces, and we prove the localization theorem of multipliers on variable exponent Lebesgue spaces. Moreover, we present a Mihlin-Hörmander type theorem for multilinear Fourier multipliers on weighted variable Lebesgue spaces and give some applications.
\end{abstract}

MSC: 42B15; 42B20; 42B25

Keywords: bilinear multiplier space; multilinear Fourier multiplier; variable exponent space; weighted estimate

\section{Introduction}

Given a non-empty open set $\Omega \subset \mathbb{R}^{n}$, we denote by $\mathcal{P}(\Omega)$ the set of exponent functions $p(x)$ such that

$$
1 \leq p_{-} \leq p_{+}<\infty
$$

where $p_{-}(\Omega):=\operatorname{essinf}\{p(x): x \in \Omega\}$ and $p_{+}(\Omega):=\operatorname{esssup}\{p(x): x \in \Omega\}$.

Let $\mathcal{P}^{0}(\Omega)$ be the set of exponent functions $p(x)$ such that

$$
0<p_{-} \leq p_{+}<\infty
$$

Given a measurable function $f$ on $\Omega$ for $1 \leq p(\cdot) \leq \infty$, we define the modular functional associated with $p(\cdot)$ by

$$
\rho_{p(\cdot), \Omega}(f)=\int_{\Omega_{\backslash \backslash \Omega}}|f(x)|^{p(x)} d x+\|f(x)\|_{L^{\infty}\left(\Omega_{\infty}\right)},
$$

where $\Omega_{\infty}$ denotes the set of points in $\Omega$ on which $p(x)=\infty$.

The variable exponent Lebesgue space $L^{p(\cdot)}(\Omega)$ is defined to be the set of Lebesgue measurable functions $f$ on $\Omega$ satisfying $\rho_{p(\cdot), \Omega}(f / \lambda)<\infty$ for some $\lambda>0$. The norm of $f$ in the space is defined by

$$
\|f\|_{L^{p(\cdot)}}=\inf \left\{\lambda>0: \rho_{p(\cdot), \Omega}(f / \lambda) \leq 1\right\} .
$$

○2014 Ren and Sun; licensee Springer. This is an Open Access article distributed under the terms of the Creative Commons Attribution License (http://creativecommons.org/licenses/by/2.0), which permits unrestricted use, distribution, and reproduction in any medium, provided the original work is properly cited. 
In the case that $p(\cdot) \in \mathcal{P}^{0}(\Omega)$, it is defined to be the set of all functions $f$ satisfying $|f(x)|^{p_{0}} \in$ $L^{q(\cdot)}(\Omega), q(x)=p(x) / p_{0} \in \mathcal{P}(\Omega)$ for some $0<p_{0}<p_{-}$(see [1]). A quasi-norm in the space is defined by

$$
\|f\|_{p(\cdot), \Omega}=\left\||f|^{p_{0}}\right\|_{q(\cdot), \Omega}^{\frac{1}{p_{0}}}
$$

We refer to [2] for an introduction to variable exponent Lebesgue spaces.

Similarly, for $p(\cdot) \in \mathcal{P}^{0}(\Omega)$ and a weight function $w$, the weighted variable exponent Lebesgue space $L^{p(\cdot)}(\Omega, w)$ (see [3]) is defined to be the set of Lebesgue measurable functions $f$ on $\Omega$ that satisfies

$$
\|f\|_{L^{p(\cdot)(w)}}:=\inf \left\{\lambda>0: \int_{\Omega}|f(x) / \lambda|^{p(x)} w(x) d x \leq 1\right\}<\infty .
$$

In this paper, we study some properties of the space of bilinear Fourier multipliers and the Mihlin-Hörmander type theorem for multilinear Fourier multipliers on weighted variable Lebesgue spaces. Specifically, let $m$ satisfy certain conditions. We discuss the $N$-linear Fourier multiplier operator $\mathrm{T}_{m}$ defined by

$$
\begin{aligned}
& \mathrm{T}_{m}\left(f_{1}, \ldots, f_{N}\right)(x) \\
& \quad=\int_{\mathbb{R}^{N n}} e^{2 \pi i\left(\xi_{1}+\cdots+\xi_{N}, x\right\rangle} m\left(\xi_{1}, \ldots, \xi_{N}\right) \hat{f}_{1}\left(\xi_{1}\right), \ldots, \hat{f}_{N}\left(\xi_{N}\right) d \xi_{1} \cdots d \xi_{N}
\end{aligned}
$$

for $x \in \mathbb{R}^{n}, f_{1}, \ldots, f_{N} \in \mathcal{S}\left(\mathbb{R}^{n}\right)[4]$.

The multilinear Fourier multipliers have been studied for a long time. In [4], Coifman and Meyer proved that $\mathrm{T}_{m}$ is bounded from $L^{p_{1}}\left(\mathbb{R}^{n}\right) \times \cdots \times L^{p_{N}}\left(\mathbb{R}^{n}\right)$ to $L^{p}\left(\mathbb{R}^{n}\right)$ for all $1<$ $p_{1}, \ldots, p_{N}, p<\infty$ with $\frac{1}{p_{1}}+\cdots+\frac{1}{p_{N}}=\frac{1}{p}$ and $m \in C^{s}\left(\mathbb{R}^{N n} \backslash\{0\}\right)$ satisfying

$$
\left|\partial_{\xi_{1}}^{\alpha_{1}} \cdots \partial_{\xi_{N}}^{\alpha_{N}} m\left(\xi_{1}, \ldots, \xi_{N}\right)\right| \leq C_{\alpha_{1}, \ldots, \alpha_{N}}\left(\left|\xi_{1}\right|+\cdots+\left|\xi_{N}\right|\right)^{-\left(\left|\alpha_{1}\right|+\cdots+\left|\alpha_{N}\right|\right)}
$$

for all $\left|\alpha_{1}\right|+\cdots+\left|\alpha_{N}\right| \leq s$, where $N \geq 2$ is an integer and $s$ is a sufficiently large integer.

Tomita [5] gave a Hörmander type theorem for multilinear multipliers. Specifically, $\mathrm{T}_{m}$ is bounded from $L^{p_{1}}\left(\mathbb{R}^{n}\right) \times \cdots \times L^{p_{N}}\left(\mathbb{R}^{n}\right)$ to $L^{p}\left(\mathbb{R}^{n}\right)$ for all $1<p_{1}, \ldots, p_{N}, p<\infty$ with $\frac{1}{p_{1}}+$ $\cdots+\frac{1}{p_{N}}=\frac{1}{p}$ and $s=\frac{N n}{2}+1$ in (1.1). Furthermore, Grafakos and Si studied the case $p \leq 1$ in [6]. The boundedness of multilinear Calderón-Zygmund operators with multiple weights was achieved by Grafakos et al. [7].

Under the Hörmander conditions, Fujita and Tomita [8] obtained some weighted estimates of $\mathrm{T}_{m}$ for classical $A_{p}$ weights. Then Li et al. [9] got some weighted results of multilinear multipliers by considering the end-point cases, using weighted Carleson measure theory and employing multilinear interpolation theory. In [10], Chen and Lu proved a Hörmander type multilinear theorem on weighted Lebesgue spaces when the Fourier multipliers were only assumed with limited smoothness. In [11], the boundedness of $\mathrm{T}_{m}$ with multiple weights satisfying condition (1.1) was given by Bui and Duong. In [12], Li and Sun got some weighted estimates of $\mathrm{T}_{m}$ with multiple weights under the Hörmander conditions in terms of the Sobolev regularity. Huang and Xu [13] obtained the boundedness of multilinear Calderón-Zygmund operators on variable exponent Lebesgue spaces. 
In this paper, we study the weighted estimates of $\mathrm{T}_{m}$ with nearly the same conditions as in [12], but on variable exponent Lebesgue spaces.

The theory of bilinear multipliers was first studied by Coifman and Meyer [14]. They considered the ones with smooth symbols. Then, Muscalu et al. achieved some new results for non-smooth symbols in [15].

The study of bilinear multipliers has experienced a big progress since Lacey and Thiele $[16,17]$ proved that $m(\xi, \nu)=\operatorname{sign}(\xi+\alpha \nu)$ are $\left(p_{1}, p_{2}, p_{3}\right)$-multipliers for each triple $\left(p_{1}, p_{2}, p_{3}\right)$ such that $1<p_{1}, p_{2} \leq \infty, p_{3}>2 / 3$ and each $\alpha \in \mathbb{R} \backslash\{0,1\}$. In [18], Kulak and Gürkanlı first studied some properties of the bilinear multiplier space. In [19], Fan and Sato proved the DeLeeuw type theorems for the transference of multilinear operators on Lebesgue and Hardy spaces from $\mathbb{R}^{n}$ to $\mathbb{T}^{n}$. In [20], Blasco gave the transference theorems from $\mathbb{R}^{n}$ to $\mathbb{Z}^{n}$. We also refer to $[21,22]$ for details.

We first give some definitions.

Definition 1.1 ([18]) Let $p_{1}(\cdot), p_{2}(\cdot) \in \mathcal{P}(\Omega), p_{3}(\cdot) \in \mathcal{P}^{0}(\Omega)$, and $m(\xi, \eta)$ be a bounded function on $\mathbb{R}^{2 n}$. Define

$$
\mathrm{B}_{m}(f, g)(x)=\int_{\mathbb{R}^{n}} \int_{\mathbb{R}^{n}} \hat{f}(\xi) \hat{g}(\eta) m(\xi, \eta) e^{2 \pi i(\xi+\eta, x)} d \xi d \eta
$$

for all $f$ and $g \in \mathcal{S}\left(\mathbb{R}^{n}\right)$.

We call $m$ a bilinear multiplier on $\mathbb{R}^{2 n}$ of type $\left(p_{1}(\cdot), p_{2}(\cdot), p_{3}(\cdot)\right)$ if there exists some $C>0$ such that $\left\|\mathrm{B}_{m}(f, g)\right\|_{p_{3}(\cdot)} \leq C\|f\|_{p_{1}(\cdot)}\|g\|_{p_{2}(\cdot)}$ for all $f$ and $g \in \mathcal{S}\left(\mathbb{R}^{n}\right)$, i.e., $\mathrm{B}_{m}$ extends to a bounded bilinear operator from $L^{p_{1}(\cdot)}\left(\mathbb{R}^{n}\right) \times L^{p_{2}(\cdot)}\left(\mathbb{R}^{n}\right)$ to $L^{p_{3}(\cdot)}\left(\mathbb{R}^{n}\right)$.

We write $\mathrm{BM}\left(\mathbb{R}^{2 n}\right)\left(p_{1}(\cdot), p_{2}(\cdot), p_{3}(\cdot)\right)$ for the space of bilinear multipliers of type $\left(p_{1}(\cdot)\right.$, $\left.p_{2}(\cdot), p_{3}(\cdot)\right)$. Let $\|m\|_{\left(p_{1}(\cdot), p_{2}(\cdot), p_{3}(\cdot)\right)}=\left\|\mathrm{B}_{m}\right\|$.

A similar function space is defined in the following.

Definition 1.2 Given a function $M$ on $\mathbb{R}^{n}$, let $m(\xi, \eta)=M(\xi-\eta)$. We say that

$$
M \in \widetilde{\mathrm{BM}}\left(\mathbb{R}^{2 n}\right)\left(p_{1}(\cdot), p_{2}(\cdot), p_{3}(\cdot)\right)
$$

if $\mathrm{B}_{M}(f, g)(x)=\int_{\mathbb{R}^{2 n}} \hat{f}(\xi) \hat{g}(\eta) M(\xi-\eta) e^{2 \pi i\langle\xi+\eta, x\rangle} d \xi d \eta$ for all $f$ and $g \in \mathcal{S}\left(\mathbb{R}^{n}\right)$ can be extended to a bounded bilinear operator from $L^{p_{1}(\cdot)}\left(\mathbb{R}^{n}\right) \times L^{p_{2}(\cdot)}\left(\mathbb{R}^{n}\right)$ to $L^{p_{3}(\cdot)}\left(\mathbb{R}^{n}\right)$.

Definition 1.3 ([2]) A function $p: \Omega \rightarrow \mathbb{R}^{1}$ is said to belong to the class $L H_{0}(\Omega)$ if

$$
|p(x)-p(y)| \leq \frac{C}{-\ln (|x-y|)}, \quad|x-y| \leq \frac{1}{2}, \quad x, y \in \Omega,
$$

where $C>0$ is independent of $x$ or $y$.

We simply write $L H_{0}$ instead of $L H_{0}\left(\mathbb{R}^{n}\right)$ if there is no confusion. We also use $C\left(\mathbb{R}^{n}\right)$ to represent the collection of all continuous functions on $\mathbb{R}^{n}$. By $C$ etc., we denote various positive constants which may have different values even in the same line. 


\section{Some results on the space $\mathrm{BM}\left(\mathbb{R}^{2 n}\right)\left(p_{1}(\cdot), p_{2}(\cdot), p_{3}(\cdot)\right)$}

Some properties of the bilinear multiplier space on variable spaces were given by Kulak and Gürkanlı [18]. Here we give some other properties.

First, we introduce the standard singular kernel.

Definition 2.1 ([2]) Given a function $K \in L_{\text {loc }}^{1}\left(\mathbb{R}^{n} \backslash\{0\}\right)$, it is called a standard singular kernel if there exists a constant $C>0$ such that:

1. $|K(x)| \leq \frac{C}{|x|^{n}}, x \neq 0$

2. $|\nabla K(x)| \leq \frac{C}{|x|^{n+1}}, x \neq 0$;

3. for $0<r<R,\left|\int_{\{r<|x|<R\}} K(x) d x\right| \leq C$;

4. $\lim _{\varepsilon \rightarrow 0} \int_{\{\varepsilon<|x|<1\}} K(x) d x$ exists.

Theorem 2.2 (Localization) Suppose that

$$
m \in \mathrm{BM}\left(\mathbb{R}^{2 n}\right)\left(p_{1}(\cdot), p_{2}(\cdot), p_{3}(\cdot)\right)
$$

$Q$ is a rectangle in $\mathbb{R}^{2 n}$ and that the Hardy-Littlewood maximal operator $\mathcal{M}$ is bounded on $L^{p_{i}(\cdot)}\left(\mathbb{R}^{n}\right)$, where $1<\left(p_{i}\right)_{-} \leq\left(p_{i}\right)_{+}<\infty, i=1,2$. Then

$$
m \chi_{Q} \in \mathrm{BM}\left(\mathbb{R}^{2 n}\right)\left(p_{1}(\cdot), p_{2}(\cdot), p_{3}(\cdot)\right)
$$

and $\left\|m \chi_{Q}\right\|_{p_{1}(\cdot), p_{2}(\cdot), p_{3}(\cdot)} \leq C\|m\|_{p_{1}(\cdot), p_{2}(\cdot), p_{3}(\cdot)}$, where $C$ is independent of $Q$.

Let $\mathrm{BM}\left(\mathbb{R}^{n}\right)(p(\cdot), p(\cdot))$ denote the space of multipliers which correspond to bounded operators from $L^{p(\cdot)}\left(\mathbb{R}^{n}\right)$ to $L^{p(\cdot)}\left(\mathbb{R}^{n}\right)$.

To prove Theorem 2.2, we need the following results in the theory of variable Lebesgue spaces.

Lemma 2.3 ([2, Theorem 5.39]) Let $T$ be a singular integral operator with a standard singular kernel K. Given $p(\cdot) \in \mathcal{P}\left(\mathbb{R}^{n}\right)$ such that $1<p_{-} \leq p_{+}<\infty$, if the Hardy-Littlewood maximal operator $\mathcal{M}$ is bounded on $L^{p(\cdot)}\left(\mathbb{R}^{n}\right)$, then for all functions $f$ that are bounded and have compact support, $\|T f\|_{p(\cdot)} \leq C\|f\|_{p(\cdot)}$, and $T$ extends to a bounded operator on $L^{p(\cdot)}\left(\mathbb{R}^{n}\right)$.

Theorem 2.4 Suppose that $m_{1} \in \mathrm{BM}\left(\mathbb{R}^{n}\right)\left(s_{1}(\cdot), p_{1}(\cdot)\right), m_{2} \in \mathrm{BM}\left(\mathbb{R}^{n}\right)\left(s_{2}(\cdot), p_{2}(\cdot)\right)$ and $m \in$ $\mathrm{BM}\left(\mathbb{R}^{2 n}\right)\left(p_{1}(\cdot), p_{2}(\cdot), p_{3}(\cdot)\right)$. Then we have

$$
m_{1}(\xi) m(\xi, \eta) m_{2}(\eta) \in \operatorname{BM}\left(\mathbb{R}^{2 n}\right)\left(s_{1}(\cdot), s_{2}(\cdot), p_{3}(\cdot)\right) .
$$

Proof For any $f$ and $g \in \mathcal{S}\left(\mathbb{R}^{n}\right)$, we have

$$
\begin{aligned}
\mathrm{B}_{m_{1} m_{2}}(f, g)(x) & =\int_{\mathbb{R}^{n}} \int_{\mathbb{R}^{n}} \hat{f}(\xi) \hat{g}(\eta) m_{1}(\xi) m(\xi, \eta) m_{2}(\eta) e^{2 \pi i\langle\xi+\eta, x\rangle} d \xi d \eta \\
& =\int_{\mathbb{R}^{n}} \int_{\mathbb{R}^{n}}\left(\left(\mathrm{~T}_{m_{1}} f\right)\right)^{\wedge}(\xi)\left(\left(\mathrm{T}_{m_{2}} g\right)\right)^{\wedge}(\eta) m(\xi, \eta) e^{2 \pi i\langle\xi+\eta, x\rangle} d \xi d \eta \\
& =\mathrm{B}_{m}\left(\mathrm{~T}_{m_{1}} f, \mathrm{~T}_{m_{2}} g\right)(x) .
\end{aligned}
$$


Therefore,

$$
\begin{aligned}
\left\|\mathrm{B}_{m_{1} m m_{2}}(f, g)\right\|_{p_{3}(\cdot)} & \leq\left\|\mathrm{B}_{m}\right\|\left\|\mathrm{T}_{m_{1}} f\right\|_{p_{1}(\cdot)}\left\|\mathrm{T}_{m_{2}} g\right\|_{p_{2}(\cdot)} \\
& \leq\left\|\mathrm{B}_{m}\right\|\left\|m_{1}\right\|_{s_{1}(\cdot), p_{1}(\cdot)}\left\|m_{2}\right\|_{s_{2}(\cdot), p_{2}(\cdot)}\|f\|_{s_{1}(\cdot)}\|g\|_{s_{2}(\cdot)} .
\end{aligned}
$$

Then we get the result.

The following is an explicit example.

Example 2.5 Suppose that $\frac{1}{p_{1}(\cdot)}+\frac{1}{p_{2}(\cdot)}=\frac{1}{p_{3}(\cdot)}, m_{1} \in \mathrm{BM}\left(\mathbb{R}^{n}\right)\left(p_{1}(\cdot), p_{1}(\cdot)\right)$ and $m_{2} \in$ $\operatorname{BM}\left(\mathbb{R}^{n}\right)\left(p_{2}(\cdot), p_{2}(\cdot)\right)$, where $p_{1}(\cdot), p_{2}(\cdot) \in \mathcal{P}\left(\mathbb{R}^{n}\right)$ and $p_{3}(\cdot) \in \mathcal{P}^{0}\left(\mathbb{R}^{n}\right)$. Then

$$
m(\xi, \eta)=m_{1}(\xi) m_{2}(\eta) \in \mathrm{BM}\left(\mathbb{R}^{2 n}\right)\left(p_{1}(\cdot), p_{2}(\cdot), p_{3}(\cdot)\right) .
$$

Proof For any $f$ and $g \in \mathcal{S}\left(\mathbb{R}^{n}\right)$, we have

$$
\begin{aligned}
\mathrm{B}_{1}(f, g)(x) & =\int_{\mathbb{R}^{n}} \int_{\mathbb{R}^{n}} \hat{f}(\xi) \hat{g}(\eta) e^{2 \pi i\langle\xi+\eta, x\rangle} d \xi d \eta \\
& =\int_{\mathbb{R}^{n}} \int_{\mathbb{R}^{n}} \hat{f}(\xi) e^{2 \pi i\langle\xi, x\rangle} \hat{g}(\eta) e^{2 \pi i\langle\eta, x\rangle} d \xi d \eta \\
& =f(x) g(x) .
\end{aligned}
$$

By Hölder's inequality [2], we have

$$
\left\|\mathrm{B}_{1}(f, g)(x)\right\|_{p_{3}(\cdot)}=\|f(x) g(x)\|_{p_{3}(\cdot)} \leq C\|f\|_{p_{1}(\cdot)}\|g\|_{p_{2}(\cdot)} .
$$

Thus $1 \in \operatorname{BM}\left(\mathbb{R}^{2 n}\right)\left(p_{1}(\cdot), p_{2}(\cdot), p_{3}(\cdot)\right)$. By Theorem 2.4, we have

$$
m(\xi, \eta)=m_{1}(\xi) m_{2}(\eta) \in \operatorname{BM}\left(\mathbb{R}^{2 n}\right)\left(p_{1}(\cdot), p_{2}(\cdot), p_{3}(\cdot)\right) .
$$

Proof of Theorem 2.2 We only consider the case $n=1$. Other cases can be proved similarly. Suppose that $Q=[a, b] \times[c, d]$. Then, for any $f$ and $g \in C_{c}^{\infty}\left(\mathbb{R}^{n}\right)$,

$$
\begin{aligned}
\mathrm{B}_{m \chi_{Q}}(f, g)(x) & =\int_{\mathbb{R}^{n}} \int_{\mathbb{R}^{n}} \hat{f}(\xi) \hat{g}(\eta) m(\xi, \eta) \chi_{Q}(\xi, \eta) e^{2 \pi i\langle\xi+\eta, x\rangle} d \xi d \eta \\
& =\int_{\mathbb{R}^{n}} \int_{\mathbb{R}^{n}} \hat{f}(\xi) \chi_{[a, b]}(\xi) \hat{g}(\eta) \chi_{[c, d]}(\eta) m(\xi, \eta) e^{2 \pi i\langle\xi+\eta, x\rangle} d \xi d \eta \\
& \left.=\mathrm{B}_{m}\left(\hat{f} \chi_{[a, b]}\right)^{\vee},\left(\hat{g} \chi_{[c, d]}\right)^{\vee}\right)(x) .
\end{aligned}
$$

Note that by (3.9) of [23], we have $\left(\hat{f} \chi_{[a, b]}\right)^{\vee}=\frac{i}{2}\left(\mathrm{M}^{a} \mathrm{HM}^{-a}-\mathrm{M}^{b} \mathrm{HM}^{-b}\right) f$, where $\mathrm{M}^{a}$ denotes the operator $\mathrm{M}^{a} f(x)=e^{2 \pi i a x} f(x)$ and $\mathrm{H}$ denotes the Hilbert transform operator. Since the Hilbert transform has a standard singular kernel, by Lemma 2.3 we have

$$
\begin{aligned}
\left\|\left(\hat{f} \chi_{[a, b]}\right)^{\vee}\right\|_{p_{1}(\cdot)} & =\frac{1}{2}\left\|\left(\mathrm{M}^{a} \mathrm{HM}^{-a} f-\mathrm{M}^{b} \mathrm{HM}^{-b} f\right)\right\|_{p_{1}(\cdot)} \\
& \leq \frac{1}{2}\left\|\mathrm{HM}^{-a} f\right\|_{p_{1}(\cdot)}+\frac{1}{2}\left\|\mathrm{HM}^{-b} f\right\|_{p_{1}(\cdot)} \\
& \leq C\|f\|_{p_{1}(\cdot)} .
\end{aligned}
$$


So

$$
\chi_{[a, b]} \in \operatorname{BM}\left(\mathbb{R}^{n}\right)\left(p_{1}(\cdot), p_{1}(\cdot)\right) .
$$

Similarly we can prove that

$$
\chi_{[c, d]} \in \mathrm{BM}\left(\mathbb{R}^{n}\right)\left(p_{2}(\cdot), p_{2}(\cdot)\right)
$$

Hence by Theorem 2.4, we get

$$
m \chi_{Q} \in \operatorname{BM}\left(\mathbb{R}^{2 n}\right)\left(p_{1}(\cdot), p_{2}(\cdot), p_{3}(\cdot)\right)
$$

and $\left\|m \chi_{Q}\right\|_{p_{1}(\cdot), p_{2}(\cdot), p_{3}(\cdot)} \leq C\|m\|_{p_{1}(\cdot), p_{2}(\cdot), p_{3}(\cdot)}$.

Next we show that the space $\widetilde{\mathrm{BM}}\left(\mathbb{R}^{2 n}\right)\left(p_{1}(\cdot), p_{2}(\cdot), p_{3}(\cdot)\right)$ is invariant under certain operators.

Theorem 2.6 Given $p_{3}(\cdot) \in \mathcal{P}\left(\mathbb{R}^{n}\right), \phi \in L^{1}\left(\mathbb{R}^{n}\right)$, if

$$
M \in \widetilde{\mathrm{BM}}\left(\mathbb{R}^{2 n}\right)\left(p_{1}(\cdot), p_{2}(\cdot), p_{3}(\cdot)\right)
$$

then

$$
\phi * M \in \widetilde{\mathrm{BM}}\left(\mathbb{R}^{2 n}\right)\left(p_{1}(\cdot), p_{2}(\cdot), p_{3}(\cdot)\right),
$$

and $\|\phi * M\|_{p_{1}(\cdot), p_{2}(\cdot), p_{3}(\cdot)} \leq C\|\phi\|_{1}\|M\|_{p_{1}(\cdot), p_{2}(\cdot), p_{3}(\cdot)}$.

Proof For any $f$ and $g \in \mathcal{S}\left(\mathbb{R}^{n}\right)$, we have

$$
\begin{aligned}
\mathrm{B}_{\phi * M}(f, g)(x) & =\int_{\mathbb{R}^{2 n}} \hat{f}(\xi) \hat{g}(\eta)\left(\int_{\mathbb{R}^{n}} M(\xi-\eta-u) \phi(u) d u\right) e^{2 \pi i\langle\xi+\eta, x\rangle} d \xi d \eta \\
& =\int_{\mathbb{R}^{n}} \int_{\mathbb{R}^{2 n}} \widehat{\mathrm{M}^{-u}} f(\xi) \hat{g}(\eta) M(\xi-\eta) e^{2 \pi i\langle\xi+\eta, x\rangle} d \xi d \eta e^{2 \pi i\langle u, x\rangle} \phi(u) d u .
\end{aligned}
$$

By Minkowski’s inequality,

$$
\begin{aligned}
\left\|\mathrm{B}_{\phi * M}(f, g)(x)\right\|_{p_{3}(\cdot)} & \leq C \int_{\mathbb{R}^{n}}\left\|\mathrm{~B}_{M}\left(\mathrm{M}^{-u} f, g\right)(x)\right\|_{p_{3}(\cdot)}|\phi(u)| d u \\
& \leq C\|M\|_{p_{1}(\cdot), p_{2}(\cdot), p_{3}(\cdot)}\|\phi\|_{1}\|f\|_{p_{1}(\cdot)}\|g\|_{p_{2}(\cdot) .}
\end{aligned}
$$

Theorem 2.7 Suppose that $p_{3} \geq 1, M \in \widetilde{\mathrm{BM}}\left(\mathbb{R}^{2 n}\right)\left(p_{1}(\cdot), p_{2}(\cdot), p_{3}\right)$ and $\phi \in L^{1}\left(\mathbb{R}^{n}\right)$. Then

$$
\begin{aligned}
m(\xi, \eta) & :=M(\xi-\eta) \hat{\phi}(\xi+\eta) \\
& \in \operatorname{BM}\left(\mathbb{R}^{2 n}\right)\left(p_{1}(\cdot), p_{2}(\cdot), p_{3}\right),
\end{aligned}
$$

and $\|m\|_{p_{1}(\cdot), p_{2}(\cdot), p_{3}} \leq\|\phi\|_{1}\|M\|_{p_{1}(\cdot), p_{2}(\cdot), p_{3}}$. 
Proof For any $f$ and $g \in \mathcal{S}\left(\mathbb{R}^{n}\right)$, we have

$$
\begin{aligned}
\mathrm{B}_{m}(f, g)(x) & =\int_{\mathbb{R}^{2 n}} \hat{f}(\xi) \hat{g}(\eta) M(\xi-\eta)\left(\int_{\mathbb{R}^{n}} \phi(y) e^{-2 \pi i\langle\xi+\eta, y\rangle} d y\right) e^{2 \pi i\langle\xi+\eta, x\rangle} d \xi d \eta \\
& =\int_{\mathbb{R}^{n}}\left(\int_{\mathbb{R}^{2 n}} \hat{f}(\xi) \hat{g}(\eta) M(\xi-\eta) e^{2 \pi i\langle\xi+\eta, x-y\rangle} d \xi d \eta\right) \phi(y) d y \\
& =\phi * \mathrm{~B}_{M}(f, g)(x) .
\end{aligned}
$$

By Young's inequality, we have

$$
\begin{aligned}
\left\|\mathrm{B}_{m}(f, g)\right\|_{p_{3}} & \leq\|\phi\|_{1}\left\|\mathrm{~B}_{M}(f, g)\right\|_{p_{3}} \\
& =\|\phi\|_{1}\left\|\mathrm{~B}_{M}\right\|\|f\|_{p_{1}(\cdot)}\|g\|_{p_{2}(\cdot) .}
\end{aligned}
$$

Thus, we get the conclusion.

Finally, we consider the necessary condition of this kind of multipliers. The bilinear classical counterpart was obtained by Hörmander [24, Theorem 3.1] and Blasco [25]. The multilinear classical one was proved by Grafakos and Torres, see [26, Proposition 5] and [27, Proposition 2.1]. And the one for multipliers on Lorentz spaces was given by Villarroya [28, Proposition 3.1]. Some of their proofs used the translation-invariant property of the classical spaces, which is, however, no longer valid on $L^{p(\cdot)}$. In the following, we prove the variable version of the necessary condition.

Theorem 2.8 (Necessary condition) Suppose that there is a non-zero continuous bounded function $M$ such that $M \in \widetilde{\mathrm{BM}}\left(\mathbb{R}^{2 n}\right)\left(p_{1}(\cdot), p_{2}(\cdot), p_{3}(\cdot)\right)$. Then

$$
\frac{1}{\left(p_{3}\right)_{+}} \leq \frac{1}{\left(p_{1}\right)_{-}}+\frac{1}{\left(p_{2}\right)_{-}}
$$

To prove the theorem, we need the following results.

Proposition 2.9 ([2, Corollary 2.22]) Fix $\Omega$ and $1 \leq p(\cdot) \leq \infty$. If $\|f\|_{p(\cdot)} \leq 1$, then $\rho(f) \leq$ $\|f\|_{p(\cdot)} ;$ if $\|f\|_{p(\cdot)}>1$, then $\rho(f) \geq\|f\|_{p(\cdot) \text {. }}$.

Proposition 2.10 ([2, Corollary 2.23]) Given $\Omega$ and $1 \leq p(\cdot) \leq \infty$, suppose $\left|\Omega_{\infty}\right|=0$. If $\|f\|_{p(\cdot)}>1$, then

$$
\begin{aligned}
\rho(f)^{1 / p_{+}} & \leq\|f\|_{p(\cdot)} \leq \rho(f)^{1 / p_{-}} . \\
\text {If } 0<\|f\|_{p(\cdot)} & \leq 1, \text { then } \\
\rho(f)^{1 / p_{-}} & \leq\|f\|_{p(\cdot)} \leq \rho(f)^{1 / p_{+}} .
\end{aligned}
$$

Lemma 2.11 Let $M \in \widetilde{\mathrm{BM}}\left(\mathbb{R}^{2 n}\right)\left(p_{1}(\cdot), p_{2}(\cdot)\right.$, $\left.p_{3}(\cdot)\right)$. If $\frac{1}{q}=\frac{1}{\left(p_{1}\right)_{-}}+\frac{1}{\left(p_{2}\right)_{-}}-\frac{1}{\left(p_{3}\right)_{+}}$, then there exists some $C>0$ such that

$$
\left|\lambda^{n} \int_{\mathbb{R}^{n}} e^{-\lambda^{2} \xi^{2}} M(\xi) d \xi\right| \leq C\|M\|_{p_{1}(\cdot), p_{2}(\cdot), p_{3}(\cdot)} \lambda^{\frac{n}{q}},
$$

when $\lambda$ is sufficiently large. 
Proof Let $\lambda>0$. Define $G_{\lambda}$ by $\widehat{G}_{\lambda}(\xi)=e^{-2 \lambda^{2} \xi^{2}}$. By a simple change of variable, one gets that

$$
\begin{aligned}
\mathrm{B}_{M}\left(G_{\lambda}, G_{\lambda}\right)(x) & =\int_{\mathbb{R}^{2 n}} e^{-2 \lambda^{2} \xi^{2}} e^{-2 \lambda^{2} \eta^{2}} M(\xi-\eta) e^{2 \pi i\langle\xi+\eta, x\rangle} d \xi d \eta \\
& =\frac{1}{2} \int_{\mathbb{R}^{2 n}} e^{-\lambda^{2} v^{2}} e^{-\lambda^{2} \mu^{2}} M(v) e^{2 \pi i\langle\mu, x\rangle} d \mu d v \\
& =\frac{C}{\lambda^{n}} e^{-\pi^{2}\left|\frac{x}{\lambda}\right|^{2}} \int_{\mathbb{R}^{n}} e^{-\lambda^{2} v^{2}} M(v) d v,
\end{aligned}
$$

where we use the fact that $G_{\lambda}(x)=\left(e^{-2 \lambda^{2} \xi^{2}}\right)^{\vee}=\frac{C}{\lambda^{n}} e^{-\frac{\pi^{2}}{2}\left|\frac{x}{\lambda}\right|^{2}}$ [29, Example 2.2.9].

Observe that

$$
\begin{aligned}
\rho_{p_{i}(\cdot)}\left(e^{-\frac{\pi^{2}}{2}\left|\frac{x}{\lambda}\right|^{2}}\right) & =\int_{\mathbb{R}^{n}} e^{-\frac{\pi^{2}}{2}\left|\frac{x}{\lambda}\right|^{2} p_{i}(x)} d x=\lambda^{n} \int_{\mathbb{R}^{n}} e^{-\frac{\pi^{2}}{2}|u|^{2} p_{i}(\lambda u)} d u \\
& \leq \lambda^{n} \int_{\mathbb{R}^{n}} e^{-\frac{\pi^{2}}{2}|u|^{2}\left(p_{i}\right)_{-}} d u=C_{\left(p_{i}\right)_{-}} \lambda^{n}
\end{aligned}
$$

where $i=1,2$.

Similarly we have

$$
\rho_{p_{i}(\cdot)}\left(e^{-\frac{\pi^{2}}{2}\left|\frac{x}{\lambda}\right|^{2}}\right) \geq C_{\left(p_{i}\right)_{+}} \lambda^{n}, \quad i=1,2 .
$$

By Proposition 2.9, we get $\left\|e^{-\frac{\pi^{2}}{2}\left|\frac{x}{\lambda}\right|^{2}}\right\|_{p_{i}(\cdot)}>1$, when $\lambda$ is sufficiently large. Thus by Proposition 2.10, we have

$$
\rho_{p_{i}(\cdot)}\left(e^{-\frac{\pi^{2}}{2}\left|\frac{x}{\lambda}\right|^{2}}\right)^{\frac{1}{\left(p_{i}\right)+}} \leq\left\|e^{-\frac{\pi^{2}}{2}\left|\frac{x}{\lambda}\right|^{2}}\right\|_{p_{i}(\cdot)} \leq \rho_{p_{i}(\cdot)}\left(e^{-\frac{\pi^{2}}{2}\left|\frac{x}{\lambda}\right|^{2}}\right)^{\frac{1}{\left(p_{i}\right)-}} .
$$

So

$$
C_{\left(p_{i}\right)_{+}} \lambda^{n /\left(p_{i}\right)_{+}-n} \leq\left\|G_{\lambda}\right\|_{p_{i}(\cdot)} \leq C_{\left(p_{i}\right)_{-}} \lambda^{n /\left(p_{i}\right)_{-}-n}
$$

where $i=1,2$.

Similarly we can get

$$
C_{\left(p_{3}\right)_{+}} \lambda^{n /\left(p_{3}\right)_{+}-n} \leq\left\|\frac{1}{\lambda^{n}} e^{-\pi^{2}\left|\frac{x}{\lambda}\right|^{2}}\right\|_{p_{3}(\cdot)} \leq C_{\left(p_{3}\right)_{-}} \lambda^{n /\left(p_{3}\right)_{-}-n} .
$$

All the inequalities above are established when the $\lambda$ is sufficiently large.

By the assumption, we have

$$
\left\|\mathrm{B}_{M}\left(G_{\lambda}, G_{\lambda}\right)\right\|_{p_{3}(\cdot)} \leq\|M\|_{p_{1}(\cdot), p_{2}(\cdot), p_{3}(\cdot)}\left\|G_{\lambda}\right\|_{p_{1}(\cdot)}\left\|G_{\lambda}\right\|_{p_{2}(\cdot) \cdot} .
$$

Now combining (2.1), (2.2), (2.3) and (2.4), we get

$$
\begin{aligned}
C_{\left(p_{3}\right)_{+}} \lambda^{\frac{n}{\left(p_{3}\right)_{+}}-n}\left|\int_{\mathbb{R}^{n}} e^{-\lambda^{2} \xi^{2}} M(\xi) d \xi\right| & \leq\left\|\frac{C}{\lambda^{n}} e^{-\pi^{2}\left|\frac{x}{\lambda}\right|^{2}}\right\|_{p_{3}(\cdot)}\left|\int_{\mathbb{R}^{n}} e^{-\lambda^{2} v^{2}} M(v) d v\right| \\
& \leq\left\|\mathrm{B}_{M}\left(G_{\lambda}, G_{\lambda}\right)\right\|_{p_{3}(\cdot)}
\end{aligned}
$$




$$
\begin{aligned}
& \leq\|M\|_{p_{1}(\cdot), p_{2}(\cdot), p_{3}(\cdot)}\left\|G_{\lambda}\right\|_{p_{1}(\cdot)}\left\|G_{\lambda}\right\|_{p_{2}(\cdot)}
\end{aligned}
$$

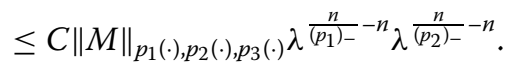

Hence

$$
\left|\lambda^{n} \int_{\mathbb{R}^{n}} e^{-\lambda^{2} \xi^{2}} M(\xi) d \xi\right| \leq C\|M\|_{p_{1}(\cdot), p_{2}(\cdot), p_{3}(\cdot)} \lambda^{\frac{n}{q}}
$$

when $\lambda$ is sufficiently large.

We are now ready to prove Theorem 2.8 .

Proof of Theorem 2.8 Assume that $\frac{1}{\left(p_{1}\right)_{-}}+\frac{1}{\left(p_{2}\right)_{-}}<\frac{1}{\left(p_{3}\right)_{+}}$. By a simple calculation, we obtain that

$$
\begin{aligned}
\mathrm{B}_{M}\left(M^{y} f, M^{-y} g\right)(x) & =\int_{\mathbb{R}^{2 n}}\left(M^{y} f\right)^{\wedge}(\xi)\left(M^{-y} g\right)^{\wedge}(\eta) M(\xi-\eta) e^{2 \pi i\langle\xi+\eta, x\rangle} d \xi d \eta \\
& =\int_{\mathbb{R}^{2 n}} T_{y} \hat{f}(\xi) T_{-y} \hat{g}(\eta) M(\xi-\eta) e^{2 \pi i\langle\xi+\eta, x\rangle} d \xi d \eta \\
& =\int_{\mathbb{R}^{2 n}} \hat{f}(\xi) \hat{g}(\eta) M(\xi-\eta+2 y) e^{2 \pi i\langle\xi+\eta, x\rangle} d \xi d \eta \\
& =\mathrm{B}_{T_{-2 y} M}(f, g)(x),
\end{aligned}
$$

where $T_{-2 y} M=M(x+2 y)$. Thus $T_{-2 y} M \in \widetilde{\mathrm{BM}}\left(\mathbb{R}^{2 n}\right)\left(p_{1}(\cdot), p_{2}(\cdot), p_{3}(\cdot)\right)$. Applying Lemma 2.11 to $T_{-2 y} M$, we get

$$
\left|\lambda^{n} \int_{\mathbb{R}^{n}} e^{-\lambda^{2} \xi^{2}} M(\xi+2 y) d \xi\right| \leq C\|M\|_{p_{1}(\cdot), p_{2}(\cdot), p_{3}(\cdot)} \lambda^{\frac{n}{q}} .
$$

Observe that $\frac{1}{q}=\frac{1}{\left(p_{1}\right)_{-}}+\frac{1}{\left(p_{2}\right)_{-}}-\frac{1}{\left(p_{3}\right)_{+}}<0$ and $M$ is continuous. By letting $\lambda \rightarrow \infty$, we have

$$
\lim _{\lambda \rightarrow \infty}\left|\lambda^{n} \int_{\mathbb{R}^{n}} e^{-\lambda^{2} \xi^{2}} M(\xi+2 y) d \xi\right|=\pi^{\frac{n}{2}}|M(2 y)|=0
$$

Since $y$ is arbitrary, we have $M=0$. This is a contradiction. Thus

$$
\frac{1}{\left(p_{3}\right)_{+}} \leq \frac{1}{\left(p_{1}\right)_{-}}+\frac{1}{\left(p_{2}\right)_{-}}
$$

\section{The Mihlin-Hörmander type estimate for multilinear multipliers on weighted variable exponent Lebesgue spaces}

Roughly speaking, in the linear case, by adding the condition that the Hardy-Littlewood maximal operator is bounded on weighted variable spaces, the results of multipliers on weighted variable spaces can be derived from the weighted multiplier theorem on classical Lebesgue spaces and the extrapolation theorem on weighted variable spaces. See, for example, [3, Theorem 4.5, Theorem 4.7], [30] and [31].

However, in the multilinear case, the method faces some challenges. One problem is that we have no multilinear extrapolation theorem on spaces with variable exponents yet, though the counterpart on classical Lebesgue spaces appeared early, see [32]. 
We give another way to get the Mihlin-Hörmander conditions for multilinear Fourier multipliers on weighted variable spaces.

First we use $Q$ to denote a cube in $\mathbb{R}^{n}$. Recall that the Hardy-Littlewood maximal operator is defined by

$$
\mathcal{M}(f)(x)=\sup _{Q \ni x} \frac{1}{|Q|} \int_{Q}|f(y)| d y .
$$

And the sharp maximal function is defined by

$$
\mathrm{M}^{\#}(f)(x)=\sup _{Q \ni x} \inf _{\mathbb{R}} \frac{1}{|Q|} \int_{Q}|f(y)-c| d y .
$$

For $\delta>0$, we also define

$$
\mathrm{M}_{\delta}(f)=\mathcal{M}\left(|f|^{\delta}\right)^{1 / \delta} \quad \text { and } \quad \mathrm{M}_{\delta}^{\#}(f)=\mathrm{M}^{\#}\left(|f|^{\delta}\right)^{1 / \delta} .
$$

For $\vec{f}=\left(f_{1}, \ldots, f_{N}\right)$ and $p \geq 1$, we define

$$
\mathcal{M}_{p}(\vec{f})(x)=\sup _{Q \ni x} \prod_{i=1}^{N}\left(\frac{1}{|Q|} \int_{Q}\left|f_{i}\left(y_{i}\right)\right|^{p} d y_{i}\right)^{1 / p} .
$$

Definition 3.1 ([33]) Given $\vec{P}=\left(p_{1}, \ldots, p_{N}\right)$ with $1 \leq p_{1}, \ldots, p_{N}<\infty$ and $1 / p_{1}+\cdots+1 / p_{N}=$ $1 / p$. Let $\vec{w}=\left(w_{1}, \ldots, w_{N}\right)$. Set

$$
v_{\vec{w}}=\prod_{i=1}^{N} w_{i}^{p / p_{i}}
$$

We say that $\vec{w}$ satisfies the $A_{\vec{P}}$ condition if

$$
\sup _{Q}\left(\frac{1}{|Q|} \int_{Q} v_{\vec{w}}(x) d x\right)^{1 / p} \prod_{i=1}^{N}\left(\frac{1}{|Q|} \int_{Q} w_{i}(x)^{1-p_{i}^{\prime}} d x\right)^{1 / p_{i}^{\prime}}<\infty .
$$

When $p_{i}=1$, then $\left(\frac{1}{|Q|} \int_{Q} w_{i}(x)^{1-p_{i}^{\prime}} d x\right)^{1 / p_{i}^{\prime}}$ is understood as $\left(\inf _{Q} w_{i}\right)^{-1}$.

We now give a Mihlin-Hörmander type theorem for multilinear Fourier multipliers on weighted variable exponent Lebesgue spaces.

Theorem 3.2 Suppose that $N n / 2<s \leq N n, m \in L^{\infty}\left(\mathbb{R}^{N n}\right)$ and

$$
\sup _{R>0}\left\|m(R \xi) \chi_{\{1<|\xi|<2\}}\right\|_{H^{s}\left(\mathbb{R}^{N n}\right)}<\infty .
$$

Set $r_{0}:=N n / s$, a series of variable indexes $p_{1}(x), \ldots, p_{N}(x) \in \mathcal{P}\left(\mathbb{R}^{n}\right)$, and $p(x) \in \mathcal{P}^{0}\left(\mathbb{R}^{n}\right)$, such that $\frac{1}{p_{1}(x)}+\frac{1}{p_{2}(x)}+\cdots+\frac{1}{p_{N}(x)}=\frac{1}{p(x)}$, where $\left(p_{j}\right)_{-}>r_{0}, j=1,2, \ldots, N$. Suppose that there are $0<q<p_{-}, r_{0}<q_{j}<\left(p_{j}\right)_{-}$such that the Hardy-Littlewood maximal operator $\mathcal{M}$ is bounded on $L^{\tilde{p}^{\prime}(\cdot)}\left(\left(w_{1} \cdots w_{N}\right)^{-q \tilde{p}^{\prime}(\cdot)}\right)$ and $L^{\tilde{p}_{j}^{\prime}(\cdot)}\left(w_{j}^{-q_{j} \tilde{p}_{j}^{\prime}(\cdot)}\right)$, where $\tilde{p}(x)=\frac{p(x)}{q}, \tilde{p}_{j}(x)=\frac{p_{j}(x)}{q_{j}}, j=1,2, \ldots, N$.

Then there exists some $C>0$ such that

$$
\left\|\mathrm{T}_{m}(\vec{f})\right\|_{L^{p(\cdot)}\left(w_{1}^{p \cdot \cdot)} \ldots w_{N}^{p(\cdot)}\right)} \leq C \prod_{i=1}^{N}\left\|f_{i}\right\|_{L^{p_{i}(\cdot)}\left(w_{i}^{p_{i}(\cdot)}\right)} .
$$


Before proving the theorem, we present some preliminary results. The following inequality is a classical result of Fefferman and Stein [34].

Proposition 3.3 ([34] Let $0<\delta<p<\infty$ and $w \in A_{\infty}$. Then there exists some constants $C_{n, p, \delta, w}>0$ such that

$$
\int_{\mathbb{R}^{n}}\left(\mathrm{M}_{\delta} f\right)(x)^{p} w(x) d x \leq C_{n, p, \delta, w} \int_{\mathbb{R}^{n}}\left(\mathrm{M}_{\delta}^{\#} f\right)(x)^{p} w(x) d x .
$$

The next result comes from Lemma 2.6 in [12]. For our purpose, we restate it in the proper way.

Proposition 3.4 ([12]) Let $1<r<\min \left\{\frac{s}{(s-1)}\right.$, $\left.\frac{2 s}{N n}\right\}$ such that $p_{0}:=r r_{0}<q_{j}, j=1, \ldots, N$. If $0<$ $\delta<p_{0} / N$, then under the assumption of Theorem 3.2, there exists some $C>0$ such that for all $\vec{f} \in L^{t_{1}}\left(\mathbb{R}^{n}\right) \times \cdots \times L^{t_{N}}\left(\mathbb{R}^{n}\right), p_{0} \leq t_{1}, \ldots, t_{N}<\infty$, we have

$$
\mathrm{M}_{\delta}^{\#}\left(\mathrm{~T}_{m} \vec{f}\right) \leq C \mathcal{M}_{p_{0}}(\vec{f}) .
$$

Proposition 3.5 ([3]) Let $X$ be a metric measure space and $\Omega$ be an open set in $X$. Assume that for some $p_{0}$ and $q_{0}$ satisfying

$$
0<p_{0} \leq q_{0}<\infty, \quad p_{0}<p_{-} \text {and } \frac{1}{p_{0}}-\frac{1}{p_{+}}<\frac{1}{q_{0}},
$$

and for every weight $w \in A_{1}(\Omega)$, there holds the inequality

$$
\left(\int_{\Omega} f^{q_{0}}(x) w(x) d \mu(x)\right)^{\frac{1}{q_{0}}} \leq c_{0}\left(\int_{\Omega} g^{p_{0}}(x)[w(x)]^{\frac{p_{0}}{q_{0}}} d \mu(x)\right)^{\frac{1}{p_{0}}}
$$

for all $(f, g)$ in a given family $\mathscr{F}$. Let the variable exponent $q(x)$ be defined by

$$
\frac{1}{q(x)}=\frac{1}{p(x)}-\left(\frac{1}{p_{0}}-\frac{1}{q_{0}}\right)
$$

Let the exponent $p(x)$ and the weight $\varrho$ satisfy that $p \in \mathcal{P}^{0}(\Omega)$ and $\mathcal{M}$ is bounded on $L^{\tilde{q}^{\prime}(\cdot)}\left(\Omega, \varrho^{-q_{0} \tilde{q}^{\prime}(\cdot)}\right)$.

Then, for all $(f, g) \in \mathscr{F}$ with $f \in L^{q(\cdot)}\left(\Omega, \varrho^{q(\cdot)}\right)$, the inequality

$$
\|f\|_{L^{q(\cdot)}\left(\Omega, \varrho^{q(\cdot)}\right)} \leq C\|g\|_{L^{p \cdot \cdot}\left(\Omega, \varrho^{p \cdot(\cdot)}\right)}
$$

is valid with a constant $C>0$.

Remark 3.6 Note that the condition $p \in \mathcal{P}(\Omega)$ in the extrapolation theorem of [3] can be released to $p \in \mathcal{P}^{0}(\Omega)$ with nearly no modification to the proof.

Proposition 3.7 ([11, Proposition 2.3]) Let $p_{0} \geq 1$ and $p_{i}>p_{0}$ for $i=1, \ldots, N$ and $1 / p_{1}+$ $\cdots+1 / p_{N}=1 / p$. Then the inequality

$$
\left\|\mathcal{M}_{p_{0}}(\vec{f})\right\|_{L^{p}\left(v_{\vec{w}}\right)} \leq C \prod_{i=1}^{N}\left\|f_{i}\right\|_{L^{p_{i}\left(w_{i}\right)}}
$$

holds if and only if $\vec{w} \in A_{\vec{P} / p_{0}}$, where $\vec{P} / p_{0}=\left(p_{1} / p_{0}, \ldots, p_{N} / p_{0}\right)$. 
Remark 3.8 When $N=1$, the conclusion above is valid. Specifically, let $p_{0} \geq 1$ and $p>p_{0}$, then $\left\|\mathcal{M}_{p_{0}} f\right\|_{L^{p}(w)} \leq C\|f\|_{L^{p}(w)}$ holds if and only if $w \in A_{p / p_{0}}$.

We are now ready to prove Theorem 3.2

Proof of Theorem 3.2 For any $f_{j} \in \mathcal{S}\left(\mathbb{R}^{n}\right), j=1, \ldots, N$, and $v \in A_{\infty}$, by Proposition 3.3 and Proposition 3.4, we have

$$
\begin{aligned}
\left\|\mathrm{T}_{m}(\vec{f})\right\|_{L^{q}(v)} & \leq\left\|\mathrm{M}_{\delta}\left(\mathrm{T}_{m}(\vec{f})\right)\right\|_{L^{q}(v)} \\
& \leq C_{n, q, \delta, v}\left\|\mathrm{M}_{\delta}^{\#}\left(\mathrm{~T}_{m}(\vec{f})\right)\right\|_{L^{q}(v)} \\
& \leq C\left\|\mathcal{M}_{p_{0}}(\vec{f})\right\|_{L^{q}(v)},
\end{aligned}
$$

where $p_{0}$ is defined as in Proposition 3.4.

Since the maximal operator $\mathcal{M}$ is bounded on $L^{\tilde{p}^{\prime} \cdot(\cdot)}\left(\left(w_{1} \cdots w_{N}\right)^{-q \tilde{p}^{\prime}(\cdot)}\right)$, by Proposition 3.5, we have

$$
\left\|\mathrm{T}_{m}(\vec{f})\right\|_{L^{p(\cdot)}\left(w_{1}^{p(\cdot)} \ldots w_{N}^{p(\cdot)}\right)} \leq C\left\|\mathcal{M}_{p_{0}}(\vec{f})\right\|_{L^{p(\cdot)}\left(w_{1}^{p(\cdot)} \ldots w_{N}^{p(\cdot)}\right)} .
$$

By Hölder's inequality,

$$
\begin{aligned}
\left\|\mathcal{M}_{p_{0}}(\vec{f})\right\|_{L^{p(\cdot)}\left(w_{1}^{p(\cdot)} \ldots w_{N}^{p(\cdot)}\right)} & \left\|\mathcal{M}_{p_{0}}(\vec{f}) w_{1} \cdots w_{N}\right\|_{L^{p(\cdot)}} \leq\left\|\prod_{i=1}^{N}\left\{\mathcal{M}_{p_{0}}\left(f_{i}\right) w_{i}\right\}\right\|_{L^{p(\cdot)}} \\
& \leq C\left\|\mathcal{M}_{p_{0}}\left(f_{1}\right) w_{1}\right\|_{L^{p^{1}(\cdot)}} \cdots\left\|\mathcal{M}_{p_{0}}\left(f_{N}\right) w_{N}\right\|_{L^{p_{N}(\cdot)}},
\end{aligned}
$$

where

$$
\mathcal{M}_{p_{0}}\left(f_{i}\right):=\sup _{Q \ni x}\left(\frac{1}{|Q|} \int_{Q}\left|f_{i}\left(y_{i}\right)\right|^{p_{0}} d y_{i}\right)^{\frac{1}{p_{0}}}, \quad i=1, \ldots, N .
$$

Since $p_{0}<q_{j}$, we can choose $u_{j}>1$ such that $p_{0} u_{j}=q_{j}$. Thus by Proposition 3.7, we get that

$$
\left\|\mathcal{M}_{p_{0}}(f)\right\|_{L^{q_{j}(w)}} \leq C\|f\|_{L^{q_{j}}(w)}
$$

is valid for all $w \in A_{u_{j}}, f \in L^{q_{j}}(w)$. Using the boundedness of $\mathcal{M}$ again, we see from Proposition 3.5 that

$$
\left\|\mathcal{M}_{p_{0}}\left(f_{j}\right)\right\|_{L^{p_{j}(\cdot)}\left(w_{j}^{p_{j}(\cdot)}\right)} \leq C\left\|f_{j}\right\|_{L^{p_{j}(\cdot)}\left(w_{j}^{p_{j}(\cdot)}\right)}, \quad j=1, \ldots, N .
$$

It follows from (3.3) that

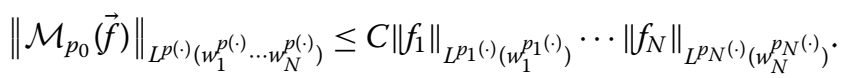

By (3.2), we obtain the desired conclusion as follows:

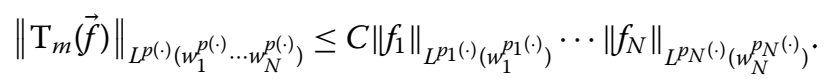


As an application of Theorem 3.2, we now consider the case when weight functions are defined by

$$
w_{j}(x)=\left[1+\left|x-x_{0}\right|\right]^{\beta_{\infty}^{j}} \prod_{k=1}^{l}\left|x-x_{k}\right|^{\beta_{k}^{j}}, \quad j=1, \ldots, N
$$

where $x_{k}$ are fixed points in $\mathbb{R}^{n}, k=1, \ldots, l$.

Corollary 3.9 Suppose that $N n / 2<s \leq N n, m \in L^{\infty}\left(\mathbb{R}^{N n}\right)$ and

$$
\sup _{R>0}\left\|m(R \xi) \chi_{\{1<|\xi|<2\}}\right\|_{H^{s}\left(\mathbb{R}^{N n}\right)}<\infty .
$$

Let the variable exponents $p_{1}(x), \ldots, p_{N}(x)$ and $p(x)$ satisfy that $\frac{1}{p_{1}(x)}+\frac{1}{p_{2}(x)}+\cdots+\frac{1}{p_{N}(x)}=\frac{1}{p(x)}$, where $1<p_{-} \leq p_{+}<\infty, r_{0}:=N n / s<\left(p_{j}\right)_{-} \leq\left(p_{j}\right)_{+}<\infty$, and $p_{j} \in L H_{0}\left(\mathbb{R}^{n}\right)$. Suppose that there exists some $R>0$ and $x_{0} \in \mathbb{R}^{n}$ such that $p_{j}(x) \equiv\left(p_{j}\right)_{\infty}=$ const for $x \in \mathbb{R}^{n} \backslash B\left(x_{0}, R\right)$, $j=1, \ldots, N$, and that

$$
\begin{aligned}
& -\frac{n}{p_{j}\left(x_{k}\right)}<\beta_{k}^{j}<\min \left\{\frac{n}{p_{j}^{\prime}\left(x_{k}\right)}, \frac{n}{N p^{\prime}\left(x_{k}\right)}\right\}, \quad k=1, \ldots, l, \\
& -\frac{n}{\left(p_{j}\right)_{\infty}}<\beta_{\infty}^{j}+\sum_{k=1}^{l} \beta_{k}^{j}<\min \left\{\frac{n}{\left(p_{j}\right)_{\infty}^{\prime}}, \frac{n}{N p_{\infty}^{\prime}}\right\}
\end{aligned}
$$

for $j=1, \ldots, N$. Then $\mathrm{T}_{m}$ is bounded from $L^{p_{1}(\cdot)}\left(w_{1}^{p_{1}(\cdot)}\right) \times \cdots \times L^{p_{N}(\cdot)}\left(w_{N}^{p_{N}(\cdot)}\right)$ to $L^{p(\cdot)}\left(w_{1}^{p(\cdot)} \ldots\right.$ $\left.w_{N}^{p(\cdot)}\right)$.

To prove Corollary 3.9, we need to define a class of weight functions, which is a special case of [3, Definition 2.7].

Definition $3.10([3])$ Let $p(\cdot) \in C\left(\mathbb{R}^{n}\right)$ and there exists $R>0$ and $x_{0} \in \mathbb{R}^{n}$ such that $p(x) \equiv$ $p_{\infty}=$ const for all $x \in \mathbb{R}^{n} \backslash B\left(x_{0}, R\right)$. A weight function $w$ of the form

$$
w=\left[1+\left|x-x_{0}\right|\right]^{\beta_{\infty}} \prod_{k=1}^{l}\left|x-x_{k}\right|^{\beta_{k}}
$$

is said to belong to the class $V_{p(\cdot)}\left(\mathbb{R}^{n}, \Pi\right)$ if

$$
-\frac{n}{p\left(x_{k}\right)}<\beta_{k}<\frac{n}{p^{\prime}\left(x_{k}\right)}, \quad k=1, \ldots, l
$$

and

$$
-\frac{n}{p_{\infty}}<\beta_{\infty}+\sum_{k=1}^{l} \beta_{k}<\frac{n}{p_{\infty}^{\prime}}
$$

We first give some lemmas that are needed to prove Corollary 3.9.

Lemma 3.11 ([2, Proposition 2.3]) Given a domain $\Omega$, if $p_{+}<\infty$, then $p(\cdot) \in L H_{0}(\Omega)$ is equivalent to assuming $r(\cdot)=1 / p(\cdot) \in L H_{0}(\Omega)$. 
Lemma $3.12\left(\left[3\right.\right.$, Remark 2.10]) For every $p_{0} \in\left(1, p_{-}\right)$, there hold the implications

$$
\varrho \in V_{p(\cdot)}(\Omega, \Pi) \quad \Longrightarrow \quad \varrho^{-p_{0}} \in V_{(\tilde{p})^{\prime}(\cdot)}(\Omega, \Pi)
$$

where $\tilde{p}(\cdot)=\frac{p(\cdot)}{p_{0}}$.

Lemma 3.13 ([3, Theorem 2.10]) Suppose that $\Omega$ is an unbounded open set of $\mathbb{R}^{n}$. Let $p(\cdot) \in L H_{0}$ satisfy $1<p_{-} \leq p_{+}<\infty$, and let there exists some $R>0$ and $x_{0} \in \mathbb{R}^{n}$ such that $p(x) \equiv p_{\infty}=$ const for $x \in \Omega \backslash B\left(x_{0}, R\right)$. If $\varrho \in V_{p(\cdot)}(\Omega, \Pi)$, then $\mathcal{M}$ is bounded on the space $L^{p(\cdot)}\left(\Omega, \varrho^{p(\cdot)}\right)$.

Then we have the following lemma.

Lemma 3.14 Let $p(\cdot) \in L H_{0}$ satisfy $1<p_{-} \leq p_{+}<\infty$. Suppose that there exists some $R>0$ and $x_{0} \in \mathbb{R}^{n}$ such that $p(x) \equiv p_{\infty}=$ const for $x \in \mathbb{R}^{n} \backslash B\left(x_{0}, R\right)$. If $\varrho \in V_{p(\cdot)}\left(\mathbb{R}^{n}, \Pi\right)$, then $\mathcal{M}$ is bounded on the space $L^{(\tilde{p})^{\prime}(\cdot)}\left(\varrho^{-q_{0}(\tilde{p})^{\prime}(\cdot)}\right)$ for all $q_{0} \in\left(1, p_{-}\right)$, where $\tilde{p}(\cdot)=\frac{p(\cdot)}{q_{0}}$.

Proof If $p(\cdot) \in L H_{0}$, then $\tilde{p}(\cdot) \in L H_{0}$. By Lemma 3.11, we have $(\tilde{p})^{\prime}(\cdot) \in L H_{0}$. And since $\varrho \in V_{p(\cdot)}\left(\mathbb{R}^{n}, \Pi\right)$, by Lemma 3.12 we know $\varrho^{-q_{0}} \in V_{(\tilde{p})^{\prime}(\cdot)}\left(\mathbb{R}^{n}, \Pi\right)$. Then it follows from Lemma 3.13 that $\mathcal{M}$ is bounded on $L^{(\tilde{p})^{\prime}(\cdot)}\left(\varrho^{-q_{0}(\tilde{p})^{\prime}(\cdot)}\right)$.

Now we are ready to prove Corollary 3.9.

Proof of Corollary 3.9 Fix some $1<q<p_{-}$. Let $q_{j}, \tilde{p}(x)$ and $\tilde{p}_{j}(x)$ be defined as in Theorem 3.2. By the assumption, we have

$$
\begin{aligned}
& -\frac{n}{p_{j}\left(x_{k}\right)}<\beta_{k}^{j}<\frac{n}{p_{j}^{\prime}\left(x_{k}\right)}, \quad k=1, \ldots, l, \\
& -\frac{n}{\left(p_{j}\right)_{\infty}}<\beta_{\infty}^{j}+\sum_{k=1}^{l} \beta_{k}^{j}<\frac{n}{\left(p_{j}\right)_{\infty}^{\prime}} .
\end{aligned}
$$

So $w_{j} \in V_{p_{j}(\cdot)}\left(\mathbb{R}^{n}, \Pi\right)$. By Lemma $3.14, \mathcal{M}$ is bounded on $L^{\left(\tilde{p}_{j}\right)^{\prime} \cdot(\cdot)}\left(w_{j}^{-q_{j}\left(\tilde{p}_{j}\right)^{\prime}(\cdot)}\right)$. Again, by the assumption, we get

$$
\begin{aligned}
& -\sum_{j=1}^{N} \frac{n}{p_{j}\left(x_{k}\right)}<\sum_{j=1}^{N} \beta_{k}^{j}<\frac{n}{p^{\prime}\left(x_{k}\right)}, \quad k=1, \ldots, l, \\
& -\sum_{j=1}^{N} \frac{n}{\left(p_{j}\right)_{\infty}}<\sum_{j=1}^{N} \beta_{\infty}^{j}+\sum_{k=1}^{l} \sum_{j=1}^{N} \beta_{k}^{j}<\frac{n}{p_{\infty}^{\prime}} .
\end{aligned}
$$

Note that the left-hand sides of (3.5) and (3.6) are equal to $-\frac{n}{p\left(x_{k}\right)}$ and $-\frac{n}{p_{\infty}}$, respectively. So $w_{1} \cdots w_{N} \in V_{p(\cdot)}\left(\mathbb{R}^{n}, \Pi\right)$.

By Lemma 3.11, we know $\frac{1}{p_{j}(\cdot)} \in L H_{0}$. Therefore, $\frac{1}{p_{(\cdot)}}=\frac{1}{p_{1}(\cdot)}+\cdots+\frac{1}{p_{N}(\cdot)} \in L H_{0}$. Thus $p(\cdot) \in$ $L H_{0}$. Now by Lemma 3.14, $\mathcal{M}$ is bounded on $L^{(\tilde{p})^{\prime}(\cdot)}\left(\left(w_{1} \cdots w_{N}\right)^{-q(\tilde{p})^{\prime}(\cdot)}\right)$. By Theorem 3.2, there exists some $C>0$ such that

$$
\left\|\mathrm{T}_{m}(\vec{f})\right\|_{L^{p(\cdot)}\left(w_{1}^{\left.p \cdot(\cdot) \ldots w_{N}^{p(\cdot)}\right)}\right.} \leq C \prod_{j=1}^{N}\left\|f_{j}\right\|_{L^{p_{j}(\cdot)}\left(w_{j}^{p_{j}(\cdot)}\right)} .
$$




\section{Competing interests}

The authors declare that they have no competing interests.

\section{Authors' contributions}

The authors completed the paper together. They also read and approved the final manuscript.

\section{Acknowledgements}

This work was partially supported by the National Natural Science Foundation of China (11371200) and the Research Fund for the Doctoral Program of Higher Education (20120031110023). The authors thank Kangwei Li for very useful discussions and suggestions.

\section{Received: 4 August 2014 Accepted: 4 December 2014 Published: 18 Dec 2014}

\section{References}

1. Tao, X, Yu, X, Zhang, H: Multilinear Calderón Zygmund operators on variable exponent Morrey spaces over domains. Appl. Math. J. Chin. Univ. Ser. B 26(2), 187-197 (2011)

2. Cruz-Uribe, DV, Fiorenza, A: Variable Lebesgue Spaces. Applied and Numerical Harmonic Analysis: Foundations and Harmonic Analysis. Birkhäuser, Heidelberg (2013)

3. Kokilashvili, VM, Samko, SG: Operators of harmonic analysis in weighted spaces with non-standard growth. J. Math Anal. Appl. 352(1), 15-34 (2009)

4. Coifman, RR, Meyer, Y: Au Delà des Opérateurs Pseudo-différentiels. Astérisque, vol. 57. Société Mathématique de France, Paris (1978). With an English summary

5. Tomita, N: A Hörmander type multiplier theorem for multilinear operators. J. Funct. Anal. 259(8), 2028-2044 (2010)

6. Grafakos, L, Si, Z: The Hörmander type multiplier theorem for multilinear operators. J. Reine Angew. Math. 668, 133-147 (2012)

7. Grafakos, L, Liu, L, Maldonado, D, Yang, D: Multilinear analysis on metric spaces. Diss. Math. 497, 121 (2014)

8. Fujita, M, Tomita, N: Weighted norm inequalities for multilinear Fourier multipliers. Trans. Am. Math. Soc. 364(12), 6335-6353 (2012)

9. Li, W, Xue, Q, Yabuta, K: Weighted version of Carleson measure and multilinear Fourier multiplier. Forum Math. (2012). do::10.1515/forum-2012-0083

10. Chen, J, Lu, G: Hörmander type theorems for multi-linear and multi-parameter Fourier multiplier operators with limited smoothness. Nonlinear Anal. 101, 98-112 (2014)

11. Bui, TA, Duong, XT: Weighted norm inequalities for multilinear operators and applications to multilinear Fourier multipliers. Bull. Sci. Math. 137(1), 63-75 (2013)

12. Li, K, Sun, W: Weighted estimates for multilinear Fourier multipliers. (2012). arXiv:1207.5111 [math.CA]

13. Huang, $A, X u$, J: Multilinear singular integrals and commutators in variable exponent Lebesgue spaces. Appl. Math. J. Chin. Univ. Ser. B 25(1), 69-77 (2010)

14. Coifman, RR, Meyer, Y: Fourier analysis of multilinear convolutions, Calderón's theorem, and analysis of Lipschitz curves. In: Euclidean Harmonic Analysis (Proc. Sem., Univ. Maryland, College Park, Md., 1979). Lecture Notes in Math., vol. 779, pp. 104-122. Springer, Berlin (1980)

15. Muscalu, C, Tao, T, Thiele, C: Multi-linear operators given by singular multipliers. J. Am. Math. Soc. 15(2), 469-496 (2002)

16. Lacey, M, Thiele, $C: L^{p}$ Estimates on the bilinear Hilbert transform for $2<p<\infty$. Ann. Math. (2) 146(3), 693-724 (1997)

17. Lacey, M, Thiele, C: On Calderón's conjecture. Ann. Math. (2) 149(2), 475-496 (1999)

18. Kulak, Ö, Gürkanlı, AT: Bilinear multipliers of weighted Lebesgue spaces and variable exponent Lebesgue spaces. J. Inequal. Appl. 2013, 259 (2013)

19. Fan, D, Sato, S: Transference on certain multilinear multiplier operators. J. Aust. Math. Soc. 70(1), 37-55 (2001)

20. Blasco, O: Bilinear multipliers and transference. Int. J. Math. Math. Sci. 2005(4), 545-554 (2005)

21. Auscher, P, Carro, MJ: On relations between operators on $\mathbf{R}^{N}, \mathbf{T}^{N}$ and $\mathbf{Z}^{N}$. Stud. Math. 101(2), 165-182 (1992)

22. Blasco, O: Notes in transference of bilinear multipliers. In: Advanced Courses of Mathematical Analysis, vol. III, pp. 28-38. World Scientific, Hackensack (2008)

23. Duoandikoetxea, J: Fourier Analysis. Graduate Studies in Mathematics. Am. Math. Soc., Providence (2001). Translated and revised from the 1995 Spanish original by David Cruz-Uribe

24. Hörmander, L: Estimates for translation invariant operators in $L^{p}$ spaces. Acta Math. 104, $93-140$ (1960)

25. Blasco, O: Notes on the spaces of bilinear multipliers. Rev. Unión Mat. Argent. 50(2), 23-37 (2009)

26. Grafakos, L, Torres, RH: Multilinear Calderón-Zygmund theory. Adv. Math. 165(1), 124-164 (2002)

27. Grafakos, L, Soria, J: Translation-invariant bilinear operators with positive kernels. Integral Equ. Oper. Theory 66(2), 253-264 (2010)

28. Villarroya, F: Bilinear multipliers on Lorentz spaces. Czechoslov. Math. J. 58(133)(4), 1045-1057 (2008)

29. Grafakos, L: Classical Fourier Analysis, 2nd edn. Graduate Texts in Mathematics, vol. 249. Springer, Heidelberg (2008)

30. Kurtz, DS: Littlewood-Paley and multiplier theorems on weighted $L^{p}$ spaces. Trans. Am. Math. Soc. 259(1), 235-254 (1980)

31. Kurtz, DS, Wheeden, RL: Results on weighted norm inequalities for multipliers. Trans. Am. Math. Soc. 255, 343-362 (1979)

32. Grafakos, L, Martell, JM: Extrapolation of weighted norm inequalities for multivariable operators and applications. J. Geom. Anal. 14(1), 19-46 (2004)

33. Lerner, AK, Ombrosi, S, Pérez, C, Torres, RH, Trujillo-González, R: New maximal functions and multiple weights for the multilinear Calderón-Zygmund theory. Adv. Math. 220(4), 1222-1264 (2009)

34. Fefferman, C, Stein, EM: $H^{p}$ Spaces of several variables. Acta Math. 129(3-4), 137-193 (1972)

10.1186/1029-242X-2014-510

Cite this article as: Ren and Sun: Multilinear Fourier multipliers on variable Lebesgue spaces. Journal of Inequalities and Applications 2014, 2014:510 